\title{
Terrestrial Magnetism
}

\author{
and \\ Atmospheric Electricity
}

VOLUME X

DECEMBER, I 905

NUMBER 4

LUFTELEKTRISCHE BEOBACHTUNGEN WÄHREND DER TOTALEN SONNENFINSTERNIS I905 AUGUST 30. IN PALMA DE MALLORCA.

VON H. EBERT.

Auf die Bedeutung elektrischer Beobachtungen während einer totalen Sonnenfinsternis haben in dieser Zeitschrift vor kurzem die Herren $/$. Elster und $H$. Geitel hingewiesen (S. I7; vergl. auch L. A. Bauer ebenda S. 2I). Die erst Genannten haben selbst,-unterstuitzt durch Mittel des Department Terrestrial Magnetism der Carnegie Institution, - eine grössere Anzahl diesbezüglicher Messungen in Palma de Mallorca angestellt, iber die sie an geeigneter Stelle berichten werden. Ich habe an gleichen Orte am Tage der Verfinsterung eine Reihe von Ionenzählungen vorgenommen mit dem von mir construierten Aspirationsapparate und zwar mit der zuletzt beschriebenen Form (Verhand1. d. Deutschen Phys. Ges. 7, 35, I 905) mit vertikal stehendem Saugrohre, welche von der Windrichtung unabhängige Werte liefert. Gleichzeitig bestimmte Herr Dr. Freiherr von und zu Aufsess mittels eines Assmann'schen Aspirations-Psychrometers die Luft-Temperatur und -Feuchtigkeit. Von Seiten des deutschen Consulates in Palma wurde uns deutschen Beobachtern eine Terrasse der Villa Formighera zur Verfïgung gestellt, welche dicht am Meeresstrande, ca. $30 \mathrm{~m}$ oberhalb desselben in einem Garten gelegen, einen sehr günstigen Beobachtungspunkt darbot; hiefür, sowie für mannigfache anderweitige Unterstützungen sind wir Herrn und Frau Consul Karl Schrader zt grossem Danke verpflichtet.

In den folgenden Tabellen bedeutet :

$E$ : die Elelstrizitätsmenge (in elektrostatischen Einheiten) des davorstehenden Zeichens, welche in I cbm in Form von Ladungen aller derjenigen Ionen enthalten ist, deren Wande- 
[Plate IV.|

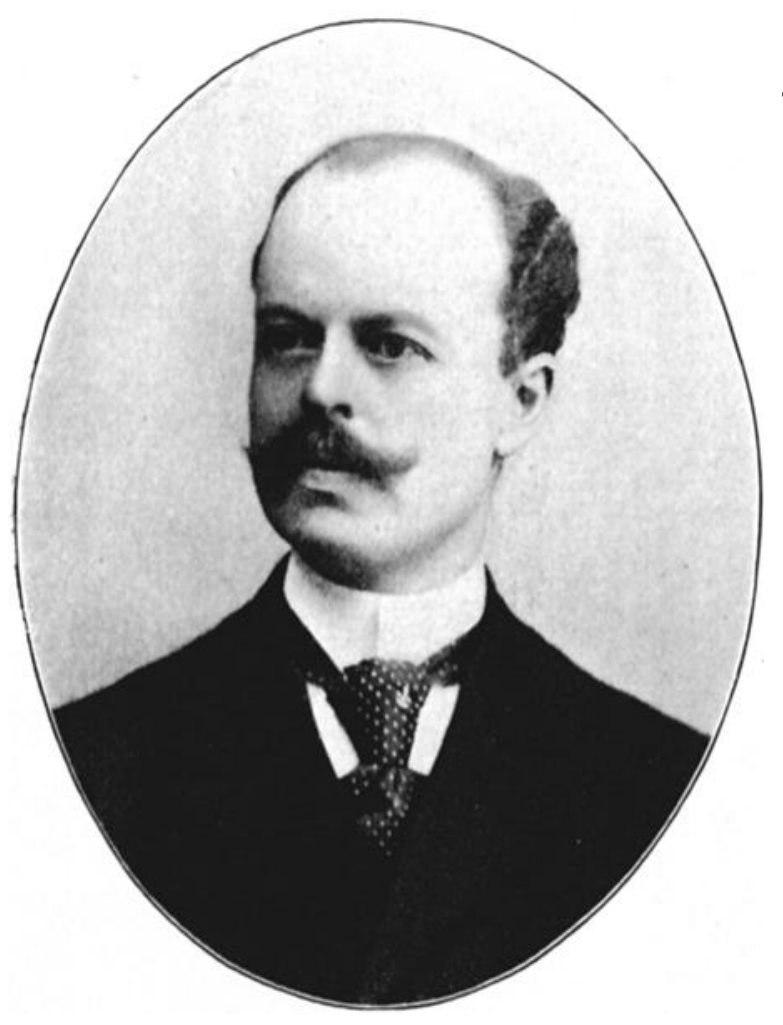

JOHANNES EDLER. 
rungsgeschwindigkeit grösser als $0.2 \mathrm{~cm} / \mathrm{sek}$ pro Volt $/ \mathrm{cm}$ Gefälle ist (das sind alle die gewöhnlichen Ionen, wie sie durch Becquerelstrahlungen und aktivierende Emanationen direkt erzeugt werden; die trägeren elektrisch geladenen Partikelchen, wie sie z. B. in der Atmosphäre durch Anlagerung dieser Ionen an Staubteilchen gebildet werden, können z. $\mathrm{T}$. durch den Apparat hindurchfliegen, ohne zur Abgabe ihrer Ladung veranlasst zu werden);

$Q$ : den Ueberschuss an positiver Ladung über die vorhandene negative Ladung in der Raumeinheit, d. i. also die negative "freie Elektrizität", welche für die Aenderung des Potentialgetälles mit der Höhe massgebend ist; es ist also: $Q=E_{+}-\mathrm{E}_{-}$; wobei die $E$ diejenigen der beiden nächstbenachbarten Beobachtungswerte sind; ein positiver Wert von $Q$ zeigt an, dass die + Ionen, ein negativer, dass die - Ionen ïberwiegen.

$p$ : den Quotienten : $\frac{E_{+}}{E_{-}}$, welcher ein Maass für die bestehende "Unipolarität" der räumlichen Ladungsdichte giebt.

$N$ : die Zahl der Ionen im $\mathrm{cbm}$ in Milliarden und

$n$ : die Ionendichte, also die Anzahl der Ionen von dem betreffenden Vorzeichen im $\mathrm{cbcm}$; bei der Ableitung der beiden letztgenannten Zahlen aus $E$ wurde die Ionenladung nach J.J. Thomson's letzter Messung zu 3.4 $\times 10^{-10}$ el. stat. Einh. angenommen ;

$t:$ die Lufttemperatur ;

$e$ : die Spannkraft des vorhandenen Wasserdampfes in mm Quecksilber, also den "Dunstdruck";

$f:$ den wahren Feuchtigkeitsgehalt in gr pro cbm Luft;

$F$ : die relative Feuchtigkeit in Procenten; die hierauf folgende Zeitangabe bezieht sich auf den Moment, in welchem die Thermometerablesungen gemacht wurden, während die in der zweiten Colonne stehenden Zeitintervalle die Dauer der Aspiration des Ionenzählers angeben; die Zeiten sind mittlere Greenwicher-Zeiten; die Nummer der Beobachtungen bezieht sich auf das Beobachtungsjournal des betreffenden Apparates. Die Windstärken wurden nach der sechsstufigen Landskala geschätzt.

Am Tage der Verfinsterung wurden ami genannten Beobachtungsorte die folgenden Zablen erhalten : 


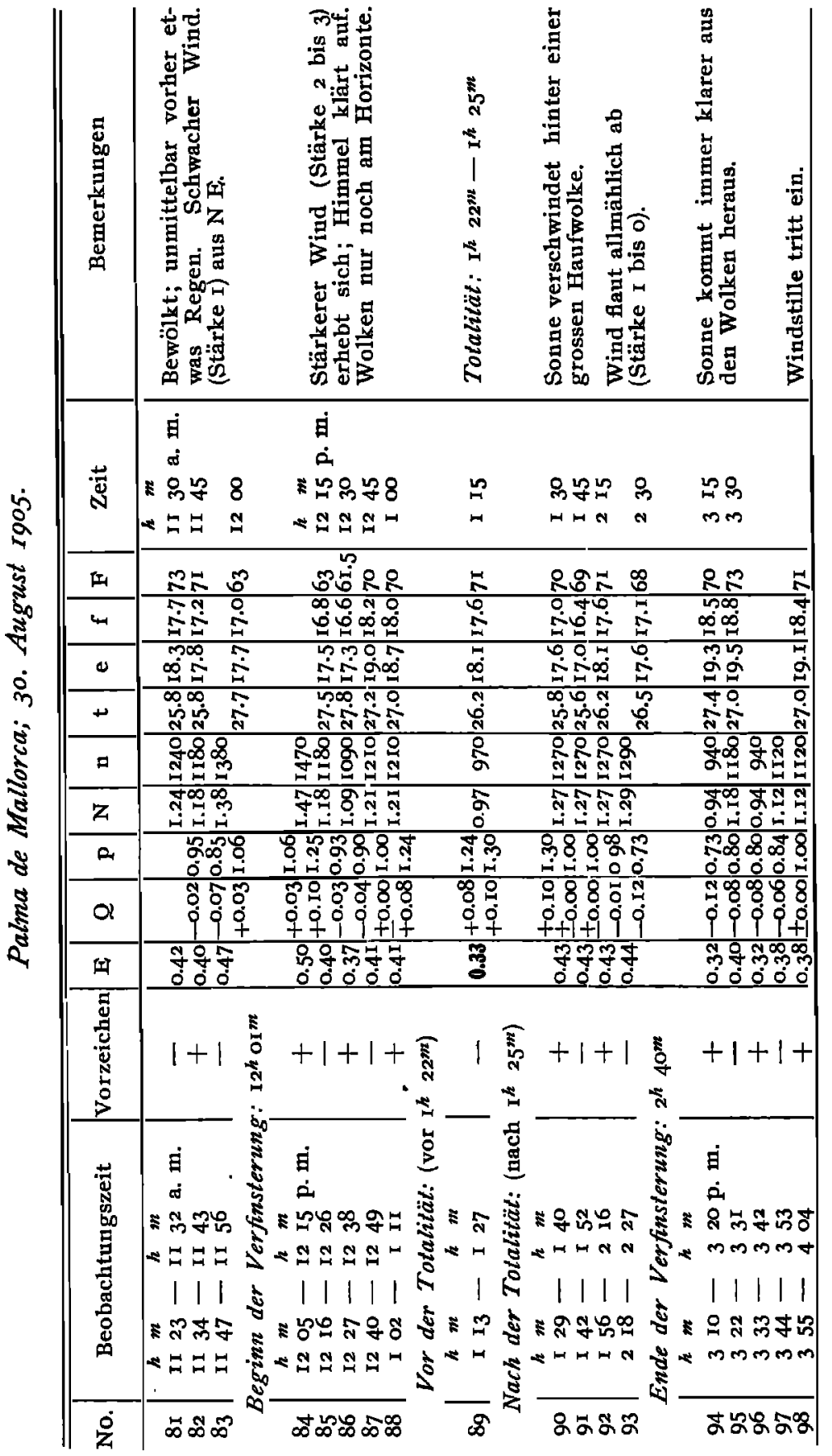


Als Mittel ergeben sich die Werte:

$$
\begin{gathered}
E_{+}=0.396 ; E_{-}=0.409 ; \text { Mittel aus } E_{+} \text {und } E=0.402 . \\
Q=-0.013 ; p=0.968 . \\
N_{+}=\text {I.16; } N_{-}=1.20 ; \text { Mittel : I.I } 8 . \\
n_{+}=\text {II60; } n_{-}=1200 ; \text { Mittel : I } 80 .
\end{gathered}
$$

An diese Zahlen möchte ich die folgenden Bemerkungen anknüpfen :

I. Die in der Raumeinheit der Luft angetroffene Ionenzahl ist an der Küste dieser balearischen Insel wesentlich kleiner, als im Innern des Continentes um dieselbe Jahreszeit und bei ähnlicher Witterungslage (allerdings in erheblich grösserer Meereshöhe). So ergaben Beobachtungen, welche unmittelbar vor der Reise nach Spanien mit demselben Apparate im Norden von München (an der Peripherie der Stadt in $540 \mathrm{~m}$ Meereshöhe) angestellt worden waren, die Mittelzahlen :

$$
\begin{gathered}
E_{+}=0.528 ; E_{-}=0.425 ; \text { Mittel }: 0.477 ; \\
\text { also: } \\
Q=+0.103 ; p=1.24 \\
N_{+}=1.55 ; N_{-}=1.25 ; \text { Mittel }: 1.40 \\
n_{+}=1550 ; n_{-}=1250 ; \text { Mittel }: 1400 ;
\end{gathered}
$$

also im Mittel etwa I.2 mal so hohe Werte als in Palma. Herr cand. phys. Max Dieckmann, der zu gleicher Zeit Ionenzählungen in dem Hochthale der Jachenau (in einer Meereshöhe von $790 \mathrm{~m}$ ) in der nördlichen Kalkalpenzone vornahm, fand (bei klarem Wetter) Werte für $E_{+}$bis zu I.7I, für $E_{-}$bis zu 0.86 .

Relativ noch niedrigere Ionenzahlen traf Herr G. Liideling, auf Helgoland im Sommer 1903 bei ruhigem Wetter an.' Er kommt, indem er seiner Beobachtungstabelle die ganz einwandfreien Messungen entnimmt, $z \mathfrak{u}$ den folgenden Mittelwerten für die niedrige, rings vom Seewasser umspülte Düne von Helgoland (2.3. bis 26 . Juni I903):

$$
\begin{gathered}
E_{+}=0.13 ; E_{-}=0.07 ; \text { Mittel : 0.10 } \\
Q=+0.06 ; p=1.86 \\
N_{+}=0.38 ; N_{-}=0.18 ; \text { Mittel }: 0.28 \\
n_{+}=380 ; n_{-}=\text {I80; Mittel: } 280 .
\end{gathered}
$$

1 G. Lüdeling, Luftelektrische und Staub-Messungen auf Helgoland 1903. Veröffentl. des $\mathrm{K}$. Preuss. Metereol. Instituts. Ergebnisse der met. Beob. in Potsdam 190r. Berlin 1904. 
während schon auf dem felsigen Oberlande folgende Mittelwerte (I9. bis 22. Juni) gefunden wurden:

$$
\begin{aligned}
& E_{+}=0.25 ; E_{-}=0.13 ; \text { Mittel } ; 0.19 \\
& Q=+0.12 ; p=1.92 \\
& N_{+}=0.74 ; N_{-}=0.38 ; \text { Mittel }: 0.56 \\
& n_{+}=740 ; n_{-}=380 ; \text { Mittel }: 560 .
\end{aligned}
$$

Dabei könnte immerhin die elektrische Leittähigkeit der Luft und damit die Geschwindigkeit, mit der gegebene Ladungen durch die Luftionen neutralisiert werden, also der Zerstreuungscoefficient, gross gewesen sein, weil für diese Grössen ausser der Ionendichte auch die Wanderungsgeschwindigkeit der Ionen massgebend ist; diese ist aber in den letzterwähnten Fällen in der reinen, vom Thaupunkte weit entfernten Seeluft gewiss gross gewesen.

Man dürfte nicht fehlgehen, wenn man den Grund für solche niederen Ionengehalte an Meereskïsten und auf Inseln in folgenden drei Momenten erblickt, auf die auch schon Herr Lïdeling selbst hingewiesen hat:

a) im Mangel an genügender mit der Atmosphäre in Communication stehender Ërdsubstanz, welche von Elster und Geitel als ein Hauptsitz des ionisierenden Principes jedenfalls der unteren Luftschichten erkannt wurde;

b) in der Erschwerung dieser Communication infolge der Durchfeuchtung des Bodens mit Meerwasser, welche insbesondere die radioaktiven Emanationen (Radium- und Thoremanation) am freien Austritte mehr oder weniger behindert;

c) in der Ionenadsorption an Wasserteilchen und Staubpartikelchen, welche die Luft an der Meeresküste immer, auch bei ganz ruhigem Wetter, reichlich erfüllen (vgl. hierüber die interessanten Staubzählungen von G. Lüdeling ${ }^{1}$ ); mit diesen Partikelchen zugleich wird auch eine grössere Anzahl der durch sie beschwerten Ionen immer wieder niedergeschlagen oder durch den Wind fortgefuihrt.

Andererseits liegen aber die auf Mallorca gefundenen Werte wesentlich höher, als die auf freier See vor und nach dem Finsternistermine im westlichen Teile des mitelländischen Meeres gefundenen Zahlen, wie die nachfolgenden Tabellen erläutern mögen. Der Beobachtungsort war die Commandobrücke der Dampfyacht

I Vergl. G. Lüdeling, Luftelektrische und Staub-Messungen an der Ostsee 1902. Veröffentl. des K. Preuss. Metereo1. Institutes. Ergebnisse der met. Beob. in Potsdam Igor. Berlin I904, sowie die oben citierten Messungen auf Helgoland. 
"Hispania" der Compagnia servizio italo-spagnuolo (General-Agentur von Semler und Gerhardt), deren Commandant Herr Capitän Antonio Serra die Aufstellung der Apparate daselbst guitigst gestattete; es wurde darauf gesehen, dass dieselben stets auf der Luvseite montiert waren.

Golfe de Lion; 24. August 1905.

$t=24.2 ; e=\mathrm{I} 7.7 ; f=\mathrm{I} 7.2 ; F=78\left(3^{h} 20 \mathrm{~m}\right.$ p. m. $)$.

\begin{tabular}{|c|c|c|c|c|c|c|c|c|}
\hline No. & $\begin{array}{c}\text { Beobachtungs- } \\
\text { zeit }\end{array}$ & $\begin{array}{c}\text { Vor- } \\
\text { zeichen }\end{array}$ & $\mathrm{E}$ & Q & $\mathbf{p}$ & $\mathbf{N}$ & n & Bemerkungen \\
\hline $\begin{array}{l}72 \\
73 \\
74 \\
75 \\
76\end{array}$ & \begin{tabular}{rrrr|}
$n$ & $m$ & $h$ & $m$ \\
2 & $33-2$ & 42 & p.m. \\
2 & $46-2$ & 55 \\
2 & $57-3$ & 05 \\
3 & $6-3$ & 15 \\
3 & $16-3$ & 25
\end{tabular} & $\frac{ \pm}{ \pm}$ & $\left|\begin{array}{l}0.094 \\
0.19 \\
0.21 \\
0.21 \\
0.19\end{array}\right|$ & $\begin{array}{l}+0.10 \\
-0.02 \\
\pm 0.00 \\
+0.02\end{array}$ & $\begin{array}{l}2.02 \\
0.90 \\
1.00 \\
1.11\end{array}$ & $\left|\begin{array}{l}0.28 \\
0.56 \\
0.62 \\
0.62 \\
0.56\end{array}\right|$ & $\mid \begin{array}{l}280 \\
560 \\
620 \\
620 \\
560\end{array}$ & $\begin{array}{l}\text { Etwas dunstig; } \\
\text { schwacher Wind aus S.W. } \\
\text { Meer völlig ruhig. }\end{array}$ \\
\hline
\end{tabular}

Barcelona, Hafen; 27. August I905.

$t=2 \mathrm{I} .6 ; e=\mathrm{I} 4.7 ; f=\mathrm{I} 4.4 ; F=76\left(\mathrm{II}^{h} 3 \mathrm{O}^{\mathrm{m}}\right.$ a. m. .

\begin{tabular}{|c|c|c|c|c|c|c|c|c|}
\hline No. & $\begin{array}{c}\text { Beobachtungs- } \\
\text { zeit }\end{array}$ & $\begin{array}{c}\text { Vor- } \\
\text { zeichen }\end{array}$ & $E$ & $Q$ & $p$ & $\mathbf{N}$ & n & Bemerkungen \\
\hline $\begin{array}{l}77 \\
78 \\
79 \\
80\end{array}$ & \begin{tabular}{|cccc}
$h$ & $m$ & $h$ & $m$ \\
II & $28-$ II & 37 & a.m. \\
II & $38-$ II & 47 \\
II & $49-$ II & 57 \\
II & $59-12$ & 08
\end{tabular} & $\frac{\bar{t}}{t}$ & $\begin{array}{l}0.22 \\
0.31 \\
0.24 \\
0.28\end{array}$ & $\begin{array}{l}+0.09 \\
+0.07 \\
+0.04\end{array}$ & $\begin{array}{l}\text { I. } 4 \mathrm{I} \\
\mathrm{I} .29 \\
\mathrm{I} . \mathrm{I}\end{array}$ & $\left|\begin{array}{l}0.65 \\
0.91 \\
0.7 \mathrm{I} \\
0.82\end{array}\right|$ & $\left|\begin{array}{l}650 \\
910 \\
710 \\
820\end{array}\right|$ & $\begin{array}{l}\text { Himmel klar, kräftiger } \\
\text { Wind (Stärke 3-4) vom } \\
\text { Meere her aus S. E. (Da } \\
\text { Sonntag ist und alle Ha- } \\
\text { fenarbeiten ruhen, so ist } \\
\text { die Iuft völlig rein.) }\end{array}$ \\
\hline
\end{tabular}

Golfe de Lion; I. September 1905.

\begin{tabular}{|c|c|c|c|c|c|c|c|c|}
\hline No. & $\begin{array}{c}\text { Beobachtungs- } \\
\text { zeit }\end{array}$ & $\begin{array}{c}\text { Vor- } \\
\text { zeichen }\end{array}$ & $\mathrm{E}$ & $Q$ & $\mathrm{p}$ & $\mathbf{N}$ & $\mathrm{n}$ & Bemerkungen \\
\hline $\begin{array}{l}99 \\
\text { I00 } \\
\text { 101 } \\
\text { 102 } \\
103 \\
104 \\
105 \\
106 \\
107 \\
108\end{array}$ & \begin{tabular}{|llll}
$h$ & $m$ & $k$ & $m$ \\
II & $47-I 1$ & 56 & am. \\
II & $58-I 2$ & 07 & p.m. \\
I2 & $09-12$ & 17 \\
I2 & I8-I2 & 26 \\
I2 & $28-12$ & 36 \\
I2 & $38-12$ & 46 \\
I2 & $48-12$ & 56 \\
I2 & $58-$ I & 07 \\
1 & $09-$ I & 17 \\
I & I9- I & 28
\end{tabular} & $\begin{array}{l}\frac{ \pm}{+} \\
\frac{ \pm}{+} \\
\frac{ \pm}{+} \\
\frac{+}{+}\end{array}$ & $\begin{array}{l}0.17 \\
0.094 \\
0.15 \\
0.15 \\
0.15 \\
0.22 \\
0.22 \\
0.22 \\
0.27 \\
0.27\end{array}$ & $\begin{array}{l}-0.08 \\
-0.06 \\
\pm 0.00 \\
\pm 0.00 \\
+0.07 \\
\pm 0.00 \\
\pm 0.00 \\
-0.05 \\
\pm 0.00\end{array}$ & $\begin{array}{l}0.55 \\
0.63 \\
1.00 \\
1.00 \\
1.46 \\
1.00 \\
1.00 \\
0.81 \\
1.00\end{array}$ & $\mid \begin{array}{l}0.50 \\
0.28 \\
0.44 \\
0.44 \\
0.44 \\
0.65 \\
0.65 \\
0.65 \\
0.79 \\
0.79\end{array}$ & $\begin{array}{l}500 \\
280 \\
440 \\
440 \\
440 \\
650 \\
650 \\
650 \\
790 \\
790\end{array}$ & $\left\{\begin{array}{l}\text { Himmel ziemlich klar, } \\
\text { nur einige Cirren } \mathrm{t}=\mathrm{I} 9.6 ; \\
\mathrm{e}=\mathrm{I} 2.3 ; \mathrm{f}=\mathrm{I} 2 . \mathrm{I} ; \mathrm{F}=7^{2} \\
\left(\mathrm{I} 2^{h} \circ 0^{m}\right) . \\
\text { Kräftiger Wind aus } \\
\text { E. N. E. } \\
\mathrm{t}=\mathrm{I} 9.7 ; \mathrm{e}=\mathrm{I} 2.6 ; \mathrm{f}=\mathrm{I} 2.4 ; \\
\mathrm{F}=73\left(\mathrm{I} \mathrm{o}^{m}\right) . \\
\mathrm{t}=\mathrm{Ig} .8 ; \mathrm{e}=\mathrm{I} 2.7 ; \mathrm{f}=\mathrm{I} 2.5 ; \\
\mathrm{F}=73\left(\mathrm{I}^{h} \mathrm{I}^{\mathrm{m}}\right) .\end{array}\right.$ \\
\hline
\end{tabular}


Vereinigt man die auf freier See erhaltenen Werte zu gemeinsamen Mitteln, so ergiebt sich:

$$
\begin{gathered}
E_{+}=0.193 ; E_{-}=0.18 \mathrm{I} ; \text { Mittel }: 0.187 \\
Q=+0.012 ; p=\text { I.07 } \\
N_{+}=0.57 ; N_{-}=0.53 ; \text { Mittel } ; 0.55 \\
n_{+}=570 ; n_{-}=530 ; \text { Mittel }: 550 .
\end{gathered}
$$

Die im Hafen von Barcelona erhaltenen Werte stehen bereits viel höher und zeigen eine deutlicher ausgeprägte Unipolarität:

$$
\begin{gathered}
E_{+}=0.295 ; E_{-}=0.230 ; \text { Mittel: } 0.262 \\
Q=+0.065 ; p=1.28 \\
N_{+}=0.87 ; N_{-}=0.68 ; \text { Mittel }: 0.78 \\
n_{+}=870 ; n_{-}=680 ; \text { Mittel }: 780 .
\end{gathered}
$$

Wenn auch bei der geringen $Z$ ahl der Einzelbeobachtungen hierauf kein grosses Gewicht zu legen ist, so kann man doch vielleicht in diesem Ansteigen der $E$-Werte und der Verschiebung des Gleichgewichtes in den Zahlen für die Ionen zu Gunsten der + Ionen bereits eine Einwirkung des Festlandes erblicken.

Die auf Palma erhaltenen Zahlen übertreffen die von mir auf freier See gefundenen Werte im Mittel um das 2. I-fache.

Es ist nicht uninteressant mit diesen Zahlen die Mittelwerte zu vergleichen, welche die Herren $L$. und $A$. Boltzmann vom $2 \mathrm{r}$. bis 3I. August Ig04 auf dem Atlantischen Ocean erhielten; sie fanden als Gesamtmittel ${ }^{1}$ :

$$
\begin{aligned}
E_{+}= & 0.391 ; E=0.272 ; \text { Mittel }: 0.332 \\
& Q=+0.119 ; p=\text { r.44 } \\
N_{+}= & \text {I.15; } N_{-}=0.80 ; \text { Mittel }: 0.97 \\
n_{+}= & \text {I } 50 ; n_{-}=800 ; \text { Mittel }: 970 .
\end{aligned}
$$

Das sind erheblich höhere Zahlen, namentlich tritt eine viel ausgesprochenere Unipolarität hervor. Die genannten Forscher hatten im Anfange ihrer Reise starken Seegang und später auffallende Störungen elektrostatischer Natur.

Nun ionisiert aber nach den Untersuchungen von $P h$. Lenard brandendes und verspritzendes Seewasser die umgebende Luft positiv. Dass von mir auf dem Meere ein so geringer Gehalt an

IVg1. A. Boltzmann, Sitz. Ber. der Wiener Akad. math. naturw. Cl. II3, Abt. II, a, 1465, 1904, und Physikal. Zeitschr. 6, 133, I905; E- daselbst entspricht unserem $E_{+}$und umgekehrt. 
+ Ionen gefunden wurde, kann daher dem Umstande zugeschrieben werden, dass das Meer die ganze Zeit über viel ruhiger war, als man es sonst im Löwengolfe anzutreffen gewohnt ist. Auf dieser positiven Elektrisierung durch den "splashing effect" scheint die auffallend hohe Unipolarität zu beruhen, die man ebenfalls wiederholt an Meereskiisten beobachtet hat, worauf schon Herr $J$. Elster früher hingewiesen hat. ${ }^{1}$

2. Sehr bemerkenswert ist die auffallend geringe Unipolarität in dem Ionengehalte. Während auf der bayerischen Hochebene im Mittel immer wenigstens O.I el. stat. Einheit mehr positive Elektrizität als negative Ladung im cbm der dem Erdboden benachbarten L,uft angetroffen wird, weicht dieser Betrag in Palma am genannten Tage im negativen Sinne von dem Nullwerte ab, was den anormalen Fall darstellt. Während im Binnenlande die Grösse $p$ normalerweise immer Werte zwischen I.I und I.3 erreicht, liegt sie hier der Einheit so nahe, dass man sagen kann, es waren etwa ebenso viel + Ionen in der untersuchten $L$ uft vorhanden wie - Ionen. Damit steht im engsten Zusammenhange der geringe Betrag des Potentialgefälles, welcher am Verfinsterungstage am Beobachtungsorte direkt konstatiert wurde. Denn der Ueberschuss der + Ionen über die - Ionen steht mit dem atmosphärischen Potentialgef älle offenbar in einem ursächlichen Zusammenhange; ein solcher Zusammenhang wird ja rücksichtlich der Aenderung des Gefälles mit der Höhe bereits durch die Poisson'sche Gleichung der Potentialtheorie festgestellt; hierdurch wïrde der obengenannte normale Ueberschuss $Q=+$ o.r einer Aenderung des Gefälles von 3.77 Volt $/ \mathrm{m}$ für jeden Meter Erhebung entsprechen, wie man sie in der Tat in der Nähe des Bodens häufig finden kann.

Man bemerkt, dass auch die auf freier See erhaltenen Werte auf eine sehr geringe Unipolarität der Ionenladungen hindeuten. Hier ist diese Tatsache noch auffallender, da ein mit der Meeresoberfläche in leitender Verbindung stehendes Schiff mit" seinen Hervorragungen, Spitzen und Tackelagen wie ein Fangkäfig auf die Ionen wirken muss; das vorhandene Potentialgef älle muss also sehr gering gewesen sein.

Man kommt zu dem Schlusse, dass auf dem ruhigen Meere ebenso wie auf der Küste jener Process abgestellt oder doch in seiner Wirksamkeit wesentlich gedämpft ist, welcher auf dem Festlande mit einem grösseren Ueberschusse an positiven Ionen $z$ uxgleich ein erheblich höheres Spannungsfeld erzeugt, als über den

1 J. Elster, Physikal. Zeitsch. 2, I13, 1900. 
oceanischen Gebieten. Der Verfasser ${ }^{1}$ glaubt diesen Prozess in einer Eigentümlichkeit des Diffusionsvorganges der Ionen und ionenerzeugenden Emanationen bei ihrer Wanderung durch die Capillaren des leitenden Erdreiches erblicken zu sollen, derzufolge (wegen der überwiegenden Adsorption der - Ionen) aus dem Boden bei der Transpiration der Bodenluft ein Ueberschuss an + Ionen in die darüber liegende freie Atmosphäre entweicht, der Boden selbst gegen die Atmosphäre also negativ geladen zurückbleiben muss; wo dieser Transpirationsprozess behindert ist, muss mit der Abnahme der frei werdenden Ionenzahl ïberhaupt, namentlich auch diese Unipolarität und damit im Zusammenhange auch der Betrag der Spannungswerte zurüickgehen.

Jedenfalls erscheint im Allgemeinen wenigstens in der Tat das Vorhandeñsein freien, vom Wasser nicht bedeckten Festlandes der Ausbildung des erdelektrischen Spannungsfeldes günstig, ja, für dieses sogar notwendig zu sein. Bekannt ist z. B., dass Gewittererscheinungen auf den kleinen oceanischen Inseln aller Klimate äusserst selten sind. ${ }^{2}$

Wenn dagegen Wind vom Lande her über die Meeresflächen streicht, so kann dieser auch jenen Ueberschuss an + Ionen mit auf die Wasserfächen hinübertragen und dadurch hier das Gefalle entsprechend erhöhen. In Folge einer derartigen Konvektion können daher auch weit entfernt vom Festlande auf dem Meere ähnliche Verhältnisse angetroffen werden, wie auf den Kontinenten.

Bei bewegter See oder beim Branden des Meeres an Küsten

1 Vgl. H. Ebert, Physikal. Zeitschr. 5, 135 und 499, 1904, sowie Meteorolog. Zeitschrift 20r, I904.

2 Es liessen sich leicht andere hierher gehörige Beispiele aus der Litteratur anfiihren; ich wähle nur wenige mit den vorliegenden Beobachtungen in engerem $\mathrm{Zu}$ sammenhange stehende aus: Herr J. Elster konstatierte bei Gelégenheit der totalen Sonnenfinsternis am 28. Mai 1900, die er von Algier aus verfolgte (Memor. Soc. d. Spettroscopisti Ital. 29. I900), ein ausgesprochenes Minimum des Potentialgefälles, unmittelbar nach der Totalität, welches er, - wie mir scheint, vollkommen mit Recht, - dadurch erklärt, "dass in die über die Erde gleitende Bahn des Mondschattens Luftmassen vom Meere her aspiriert werden, die von den fribheren, am Beobachtungsorte befindlichen elektrisch different waren." Die Verschiedenheit bestand in elektrischen Eigenschaften der aspirierten Luft, welche das Gefälle herabsetzten.

Dieses Beispiel zeigt übrigens wie überaus wichtig es wäre, wenn einmal an ruhigen und elektrisch normalen Tagen luftelektrische Messungen auf einem genügend störungsfreien Fahjzeuge un ternommen würden, welches sich von der Küste entfernt und dieser wieder nähert unter Mitwirksamkeit einer sekundierenden Landstation, mit deren Instrumenten das Instrumentarium des Fahrzeuges vorher und nachher zu vergleichen wäre. Fist derartige systematisch durchgeführte Untersuchungen könnten die wichtige Frage ihrer völligen Klärung entgegenführen. 
kann der "Lenardeffect" innerhalb der untersten Schichten der Atmosphäre ein hohes Potentialgefälle erzeugen.

Endlich ist bei allen Betrachtungen der erdelektrischen Zustände die Wirksamkeit des grossen regelmässigen Cirkulationssystems in der Atmosphäre nicht zu vergessen. Dieses 'kann in den Tropen einen grossen Vorrat an Ionen mit einem Ueberschusse an + Ionen in die Höhe heben und diesen in höhere Breiten übertragen, so dass in diesen immer ein gewisser Vorrat nicht gebundener + Ionen vorhanden ist, der bei seiner langsamen Diffusion in tiefere Regionen das normale luftelektrische Gefälle auf lange Dauer hinaus aufrecht zu erhalten vermag und für dieses gewissermassen einen Grundstock liefert, dem sich die lokalen und an die Erdoberfläcle selbst gebundenen sekundären Erscheinungen überlagern.

3. Was nun endlich den Einfluss der Verfinsterung der Sonne selbst betrifft, so durfte hier von vornherein nur wenig erwartet werden. Wie die Bemerkungen am Rande der ersten Tabelle zeigen, war das Wetter sehr wechselnd. Am Morgen des 30. Augusts bedeckte in Palma eine dichte Wolkendecke den ganzen Himmel; geyen II Uhr fing es sogar schwach zu regnen an, so dass alle Hoffnung von den Phänomenen der Totalität etwas zu sehen, dahinschwand. Um Mittag aber riss der Wolkenschleier auf, so dass der Beginn und das Fortschreiten der Verfinsterung verfolgt werden konnte. Unterdessen hatte sich ziemlich heftiger Wind erhoben und neue Wolken zogen heran. Zur Zeit der Totalität stand die Sonne gerade noch in einer Wolkenlïcke, fünf Minuten nachher war sie hinter einer dichten Cumuluswolke verschwunden. Erst nach dem Ende der Verfinsterung trat die Sonne wieder hervor, während unterdessen der Wind (der auch seine Richtung gewechselt hatte) abflaute. Es ist klar, dass bei solch' unbeständigen Witterungsbedingungen das Vorbeiziehen des immerhin schmalen Schattenkegels des Mondes nur einen untergeordneten Einfluss ausüben konnte, der vielleicht deutlich hervorgetreten wäre, wenn der betreffende Tag ein luftelektrisch weniger gestörter gewesen wäre. Es lässt sich demzufolge auch kein Gang, weder in den luftelektrischen noch in den meteorologischen Daten erkennen und wo ein solcher angedeutet ist, dürfte er weit eher durch die geschilderte zeitliche Aenderung der allgemeinen Wetterlage als durch die Bedeckung der Sonnenscheibe bedingt sein. Nur dem während der Totalität selbst erhaltenen Werte glaube ich eine gewisse Bedeutung beimessen zu sollen. Herr Prof. Dr. H. Geitel, 
der mit einem Aspirationsapparate gleicher Construktion (beide von der Firma Gïnther \& Tegetmeyer in Braunschweig bezogen) [aber mit dem a. a. O. erwähnten Hilfskondensator, der von dem genaunten Forscher ausser den Ionendichten auch die spezifschen Wanderungsgeschwindigkeiten mit dem Apparate bestimmt wurden] wie ich selbst arbeitete, und ich hatten die Verabredung getroffen, dass wälırend der Totalität Herr H. Geitel die Zahl der positiven, ich aber diejenige der negativen Ionen bestimmen wollte, dergestalt, dass die beiden Ergebnisse sich ergänzen sollten. Man sieht nun bei dem Vergleiche des bei der 89. Beobachtung erhaltenen Wertes mit den zeitlich benachbarten Werten (No. 85, 87 und 91, 93), dass sich während oder unmittelbar nach der Totalität die Zahl der von dem Apparate angezeigten Ionen negativen Zeichens merklich vermindert hat (etwa im Verhältnisse von $4 \mathrm{zu} 5$ ).

In Wirklichkeit könnte das Herabgehen der Zahlen für die - Ionen noch wesentlich grösser gewesen sein als es durch dieses Verhältniss zum Ausdruck kommt. Wie man sieht, erstreckt sich das Beobachtungsintervall über I4 Minuten, während die Totalität ja nur 3 Minuten dauerte; es könnte also sein, dass der Einfluss der letzteren verwischt wäre durch die Zustände, welche namentlich vor der Totalität statthatten.

Um diese auffallende Erscheinung zu erklären, könnte man etwa an Folgendes denken: Durch das Eintreten des Mondschattens in die Atmosphäre tritt daselbst eine Abkühlung ein. Von dieser lässt zwar das Aspirationsthermometer nur wenig erkennen; (das gleichmässige Herabgehen der Temperatur dürfte eher dem Herannahen des Wolkenschattens und der zunehmenden allgemeinen Trübung zuzuschreiben sein). Ein nicht aspiriertes, aber ebenfalls vor der direkten Sonnenstrahlung geschütztes anderes Thermometer ergab einen Temperatursturz von rund 8 Grad beim Eintreten der Totalität. Jedenfalls können in den vom Mondschatten erreichten Partieen des Luftmeeres partielle Condensationen des Wasserdampfes stattgefunden haben, die freilich schnell wieder aufgehoben wurden, so dass diese vorübergehenden Condensationen das Aspirations-Psychrometer unberuhrt lassen konnten. Wie bekannt sind nun gerade die Ionen besonders geeignet als Kerne bei der Condensation zı dienen. Eine grössere Anzahl derselben kann sich also vorübergehend mit Wasserdampf beladen haben, wodurch ihre Beweglichkeit erheblich herabgesetzt werden musste. Jetzt konnte eine grössere Zahl dieser Ionen durch das elektrische Spannungsfeld des Apparates hindurchschlïpfen, der, 
wie oben bereits erwähnt, mit Sicherheit nur Ionen einfängt, deren Wanderungsgeschwindigkeit von der Ordnung der gewöhnlichen "Gasionen" ist. Ausgeschlossen ist natürlich nicht, dass sich auch direkt die Zahl der Ionen selbst vermindert hat.

Wir hätten hier somit in der Tat einen gewissen Einfluss der Sonnenverfinsterung vor uns, welcher sich auf die Zah1 der Ionen wie auf ihre Wanderungsgeschwindigkeit erstreckte; der letztere wäre freilich nur ein indirekter, durch die Abkiihlung der beschatteten A tmosphäre bedingter.

Die Wirkung ist nicht unähnlich derjenigen, welche beim Einbrechen der Nacht häufig beobachtet wird. Auch hierbei gehen die Ionenmengen in der Luft zurück, die Wanderungsgeschwindigkeiten werden kleiner, wie denn auch die Zerstreuungswerte namentlich bei positiver Ladung des Zerstreuungskörpers in diesem Falle entsprechend zurückzugehen pflegen.

Mïnchen, September roo5. 